\title{
NECESSIDADE DE META-AVALIAÇÃO PARA A AUTOAVALIAÇÃO INSTITUCIONAL: CPA DO SENAC/SC
}

http://dx.doi.org/10.5902/2318133865887

\author{
Melissa Maria de Souza Zimmermann ${ }^{1}$ \\ Lourdes Alves ${ }^{2}$
}

\begin{abstract}
Resumo
O objetivo deste trabalho foi identificar se integrantes de comissões próprias de avaliação de Faculdade do Senac/SC são preparados para a realização do processo de autoavaliação institucional e se entendem como viável a realização da meta-avaliação nesse processo. A pesquisa utilizou-se de análise documental e entrevistas estruturadas, envolvendo duas faculdades do Senac/SC. Quanto aos resultados evidenciou-se a necessidade de capacitação dos integrantes das comissões, bem como foi identificada a viabilidade de inclusão da meta-avaliação no processo da autoavaliação institucional.

Palavras-chave: avaliação; meta-avaliação; autoavaliação institucional; CPA.
\end{abstract}

\section{NEED FOR A METAL-EVALUATION FOR INSTITUTIONAL SELF-EVALUATION: OEC OF SENAC/SC}

\section{Abstract}

The objective of this work is to identify if the members of own evaluation commissions are prepared to carry out the institutional self-assessment process and if they find it feasible to use methodologies that allow the inclusion of the meta-assessment in this process. The research was carried out through a qualitative approach, using bibliographic research, document analysis and structured interviews, involving two of the faculties of Senac/SC. As for the results, the need for training members of the comissions was evident, as well as the feasibility of including the metaassessment in the institutional self-assessment process.

Key-words: evaluation; meta-evaluation; institutional self-assessment; OEC.

\footnotetext{
1 Universidade Federal de Santa Catarina, Brasil. E-mail: melissazm@gmail.com.
}

2 Universidade Federal de Santa Catarina, Brasil. E-mail: loual@lourdesalves.com.br. 


\section{Introdução}

esde 1996 o MEC desenvolve ações relacionadas a avaliação institucional. No intuito de aperfeiçoar os processos de avaliação o MEC criou, em 2003, a Comissão Especial da Avaliação da Educação Superior, com a finalidade de analisar o sistema de avaliação vigente e propor alternativas para melhorá-lo. Os estudos dessa Comissão resultaram no documento Sistema Nacional de Avaliação da Educação Superior (Sinaes), bases para uma nova proposta de avaliação da educação superior (Inep, 2004), cuja regulamentação deu-se por intermédio da lei n. 10.861/2004 (Brasil, 2004).

O Sinaes propõe três principais processos de avaliação: avaliação institucional, que se subdivide em avaliação externa e autoavaliação; avaliação de cursos de graduação e o exame nacional de desempenho dos estudantes - Enade. Considerando a autoavaliação como ponto de partida para avaliação institucional, o art. 11, a lei do Sinaes determina que as instituições de educação superior (IES) constituam a Comissão Própria de Avaliação - CPA - formada por representantes de todos os segmentos da comunidade acadêmica: professores, alunos, egressos, servidores técnico-administrativos e por representantes da sociedade civil organizada. A CPA é encarregada da autoavaliação institucional e de relatar os resultados ao Inep (Brasil, 2004).

A autoavaliação pode proporcionar às IES a possibilidade de reflexão sobre suas próprias perspectivas e seus planejamentos futuros, considerando o contexto real e atual em que se encontra. Por isso, "deve ser conduzida por agentes internos da instituição e procurar o envolvimento e a legitimação de toda a comunidade universitária" (Inep, 2015, p. 31). Nesse sentido, para o aprimoramento contínuo do processo avaliativo e para que 0 mesmo possa trazer os resultados esperados, pode-se aplicar a avaliação da avalição.

A meta-avaliação é o processo de delinear, obter e aplicar informações descritivas e informações de julgamento sobre a "utilidade, viabilidade, adequação e precisão de uma avaliação" (Stufflebeam, 2000, p. 183), a fim de orientar a avaliação e relatar publicamente seus pontos fortes e fracos. Além disso, as meta-avaliações são do interesse público e profissional para assegurar que as avaliações forneçam conclusões e orientações sólidas.

$\mathrm{O}$ documento que originou a lei do Sinaes remete à necessidade de realização de meta-avaliação, tanto pelas próprias IES, quanto pelo MEC:

Os processos de avaliação interna e os da externa devem ser constantemente avaliados, tanto pelas próprias IES quanto pelo MEC. As análises dos processos de avaliação e as recomendações específicas daí derivadas devem fazer parte dos respectivos relatórios. De modo especial, cada novo ciclo avaliativo deve levar em conta os acertos e eventuais equívocos do processo anterior. Por sua vez, a Conaes fará suas recomendações para o contínuo aperfeiçoamento dos processos de avaliação, buscando verificar questões como a sua adequação à complexidade institucional e à diversidade do sistema, a utilidade das recomendações para o aperfeiçoamento das instituições e a melhoria da qualidade acadêmica, a viabilidade dos métodos e instrumentos utilizados, a justeza e a confiabilidade dos resultados, entre outros. (Inep, 2004, p. 110) 
Nessa perspectiva, é fundamental que os agentes do processo sejam capacitados e orientados para que possam exercer com excelência o papel de avaliadores, buscando atingir os objetivos da autoavaliação e aprimorando o processo a partir da análise dos resultados.

\section{A autoavaliação institucional}

A partir da implementação da lei do Sinaes as instituições iniciaram ou reorganizaram seus processos de autoavaliação a fim de atender a legislação e também de buscar a melhoria contínua de seus processos, por meio do autoconhecimento. Segundo o documento base do Sinaes,

as funções mais importantes da autoavaliação permanente são a de produzir conhecimentos, pôr em questão a realização das finalidades essenciais, identificar as causalidades dos problemas e deficiências, aumentar a consciência pedagógica e a capacidade profissional dos professores, tornar mais efetiva a vinculação da instituição com o entorno social e a comunidade mais ampla, julgar acerca da relevância científica e social de suas atividades e seus produtos, prestar contas à sociedade, justificar publicamente sua existência e fornecer todas as informações que sejam necessárias ao conhecimento do Estado e da população. (Inep, 2004, p. 96)

Ou seja, além de proporcionar e fundamentar a emissão de juízo de valor, a proposta da autoavaliação veio para mudar a cultura avaliativa das instituições no sentido de articular ações de melhoramento de toda a comunidade acadêmica e da instituição. Por isso, teve como premissa o fato de mudar a cultura institucional, instituindo processos permanentes de uma avaliação focada nas estruturas e nas ações institucionais.

A Nota técnica n. 65/2014, que estabelece o roteiro para a autoavaliação institucional, menciona que

a autoavaliação, em consonância com o Plano de Desenvolvimento Institucional (PDI) da IES, deve ser vista como um processo de autoconhecimento conduzido pela Comissão Própria de Avaliação (CPA), mas que envolve todos os atores que atuam na instituição, a fim de analisar as atividades acadêmicas desenvolvidas. É um processo de indução de qualidade da instituição, que deve aproveitar os resultados das avaliações externas e as informações coletadas e organizadas a partir do PDI, transformando-os em conhecimento e possibilitando sua apropriação pelos atores envolvidos. Afinal, as ações de melhoria a serem implementadas pela instituição dependem de sua própria compreensão, de seu autoconhecimento. (Brasil, 2014, p. 2)

Portanto, é por meio da participação ampla dos atores universitários que se pode garantir um processo de reflexão coletiva e colaborativa trazendo conhecimentos, interpretações e compreensões que resultem, de fato, em melhorias e transformações nas instituições. 


\section{A CPA e os avaliadores}

A Comissão Própria de Avaliação foi instituída pelo art. 11 da lei do Sinaes. As instituições, portanto, passaram a nomear suas próprias comissões, responsáveis pela autoavaliação institucional, cujos membros representam os segmentos da comunidade universitária - docente, discente e técnico-administrativo - e da sociedade civil organizada. A legislação veda a existência de maioria absoluta por parte de qualquer um dos segmentos representados, no entanto, estes podem ser eleitos ou nomeados observando regulamentação própria da IES. Além disso, esta comissão deve possuir um coordenador, membro da comunidade universitária que tenha experiência no processo de avaliação do Ensino Superior (Brasil, 2014).

Embora, o papel da CPA seja instrumentalizar a gestão, colaborando com o planejamento institucional e sua revisão permanente, os maiores desafios para as IES estão na escolha de seus membros; no equilíbrio dos segmentos da comunidade acadêmica e da sociedade civil organizada na sua composição; no envolvimento da comunidade universitária nos processos avaliativos; na autonomia da comissão e na dificuldade na elaboração de uma cultura avaliativa nas instituições de educação superior (Sordi, 2011).

Segundo Souza e Kipnis (2016) é fundamental que os membros das CPA tenham formação consolidada para qualificar o processo que precisam desenvolver. Além disso é necessário que os atores da autoavaliação sejam "isentos e reconhecidos por seu compromisso ético e técnico e, principalmente, que sejam legitimados pela comunidade" (p. 7). No entanto, a gestão da instituição precisa estar, de fato, comprometida com a avaliação no sentido de respaldar todo esse processo, considerando os aspectos sociais que o envolve. Sendo assim, os avaliadores, responsáveis pela autoavaliação, precisam compreender que

a autoavaliação é tão complexa como a própria Avaliação Institucional em sua completude, uma vez que é capaz de acender na comunidade acadêmica questões políticas e filosóficas sobre sua própria realidade, possibilitando a (re)elaboração da identidade institucional. Por isso, a necessidade de que os membros das CPA compreendam e assumam o papel social que lhes cabe nesse processo e que prescinde das duas concepções da avaliação para dar conta da singularidade das instituições de ensino, ou melhor, da singularidade da educação. (Souza; Kipnis, 2016, p. 7)

Com o papel de definir os membros das CPA, as IES têm diante de si o desafio de nomear representantes que possam desempenhar com excelência a função de avaliadores, trazendo as respostas necessárias para a instituição e colaborando com a melhoria contínua dos processos educacionais a fim de atingir todos os objetivos da própria autoavaliação. Para tanto, a gestão deve caminhar lado a lado com a CPA, subsidiando sua preparação e corroborando com suas ações. 


\section{Considerações sobre meta-avaliação}

Scriven (1991) define meta-avaliação como avaliação da avaliação, ou seja, é o olhar sobre o processo avaliativo no intuito de assegurar a qualidade da avaliação, o qual é realizado por meio de critérios, com acompanhamento e revisão sistemática do processo.

O documento que embasou a lei do Sinaes apresentou a necessidade da metaavaliação, considerando-a como "retroalimentação do sistema", apontando que "cada novo ciclo avaliativo deve levar em conta os acertos e eventuais equívocos do processo anterior" (Inep, 2004, p. 110). Portanto, nota-se que a meta-avaliação proporciona mecanismos para aferir a importância das ações avaliativas desenvolvidas, além de apontar a relevância, o mérito e os fatores que necessitam de melhorias. Sendo assim, "a meta-avaliação transforma-se em uma atividade essencial para o fortalecimento de uma cultura de avaliação consolidada por um referencial teórico consistente e por uma prática não punitiva, que visa ao desenvolvimento da IES" (Gimenes, 2007, p.229).

Stufflebeam (2000) apresenta ainda dois tipos de meta-avaliação: as formativas e as somativas. As meta-avaliações formativas são realizadas no planejamento de uma avaliação ou enquanto ela está em andamento, ajudando os avaliadores a planejar, conduzir, melhorar, interpretar e relatar seus estudos de avaliação. Já as meta-avaliações somativas são conduzidas após a conclusão de uma avaliação e ajudam o público a ver os pontos fortes e fracos de uma avaliação e a julgar seu mérito e valor de acordo com os padrões de boas práticas de avaliação.

Contudo, para ambos os tipos de meta-avaliação, há que se levar em consideração a preparação dos avaliadores, já que "não se presume como condição necessária a metaavaliação ser realizada por meta-avaliadores externos, pois ela pode ser realizada pelos mesmos agentes da avaliação primária" (Davok, 2006, p. 86), embora órgãos especializados no assunto recomendem a realização por avaliadores externos, evitando contratempos e interposições advindas de políticas, jogos de interesses ou parcialidades.

$O$ fato é que se forem emitidos relatórios defeituosos sem serem expostos a metaavaliações sólidas, o público-alvo da avaliação pode tomar decisões erradas com base nos resultados errados (Stufflebeam, 2000). Ou seja, tão importante quanto o processo de meta-avaliação é a capacitação de avaliadores e meta-avaliadores para garantir a eficiência e eficácia das avaliações, em especial, da autoavaliação institucional, que busca a melhoria contínua das instituições para garantir a qualidade da educação superior no país.

\section{Meta-avaliação e os atributos das práticas de avaliação educacional}

O Comitê Conjunto de Padrões para Avaliação Educacional - Join Committee on Standards Educational Evaluation - JCSEE - é a fonte oficial de padrões de avaliação nos Estados Unidos e no Canadá. O JCSEE foi formado em 1975 como uma coalizão de associações profissionais preocupadas com a qualidade das práticas de avaliação. $A$ comissão é composta por representantes de organizações profissionais e especialistas no assunto, com a tarefa de rever, atualizar, desenvolver e disseminar padrões para atender as necessidades do campo da avaliação. 
A associação ao comitê inclui representação da comunidade educacional, avaliadores profissionais e especialistas no assunto, cada um com interesse no uso e na prática da avaliação. O primeiro conjunto de normas, The Program Evaluation Standards, foi publicado em 1981. Desde então o comitê continuou a revisar e atualizar os padrões originais, bem como a desenvolver novos padrões para áreas especializadas de avaliação educacional. Hoje o JCSEE é a fonte oficial de padrões para avaliação de programas na América do Norte e fornece padrões aplicáveis às avaliações realizadas em ambientes educacionais e não educacionais. Os padrões de avaliação do programa também foram adotados oficialmente por outras comunidades de avaliação em todo o mundo.

Segundo Galvão et al (2014) os critérios do JCSEE são formados por trinta tópicos, fundamentados com suas respectivas definições, diretrizes, apontamentos e erros comuns e exemplos de aplicabilidade. Além disso, com base na discussão sobre diretrizes, o JCSEE desenvolveu o conceito que aponta que "a qualidade de um estudo avaliatório pode ser determinada por sua utilidade, viabilidade, propriedade e precisão" (Galvão et al, 2014, p. 7). A partir desta definição foram agrupadas as trinta diretrizes conforme sua contribuição para cada um desses quatro atributos. A última revisão dos padrões de avaliação apresentada pelo JCSEE, em 2011, incluiu o atributo de responsabilização. Desta forma os padrões de avaliação educacional passaram a contemplar cinco atributos: utilidade, viabilidade, adequação, precisão e responsabilização, com os trinta critérios distribuídos entre eles (JCSEE, 2011).

Embora todos os atributos apresentados pelo JCSEE sejam de elevada importância para o processo avaliativo, neste trabalho serão utilizados os atributos de precisão e responsabilização pela sua aderência ao processo de autoavaliação institucional e também pela ligação com a função dos avaliadores.

Quadro 1 -

Padrões de avaliação do JCEE aderentes à meta-avalição.

\begin{tabular}{|c|c|}
\hline & Padrões de precisão \\
\hline $\begin{array}{l}\text { Os padrões de precisão visam } \\
\text { aumentar a confiabilidade e a } \\
\text { veracidade das representações, } \\
\text { proposições e descobertas da } \\
\text { avaliação, especialmente aquelas } \\
\text { que apoiam interpretações e } \\
\text { julgamentos sobre a qualidade. }\end{array}$ & $\begin{array}{l}\text { A1 Conclusões e decisões justificadas. As conclusões e } \\
\text { decisões da avaliação devem ser explicitamente justificadas } \\
\text { nas culturas e contextos em que têm consequências. } \\
\text { A2 Informações válidas. As informações de avaliação } \\
\text { devem servir aos propósitos pretendidos e apoiar } \\
\text { interpretações válidas. } \\
\text { A3 Os procedimentos de avaliação de informações } \\
\text { confiáveis devem fornecer informações suficientemente } \\
\text { confiáveis e consistentes para os usos pretendidos. } \\
\text { A4 Descrições explícitas do programa e do contexto. As } \\
\text { avaliações devem documentar os programas e seus } \\
\text { contextos com detalhes e escopo adequados para os fins } \\
\text { da avaliação. } \\
\text { A5 As avaliações de gerenciamento de informações devem } \\
\text { empregar métodos sistemáticos de coleta, revisão, } \\
\text { verificação e armazenamento de informações. } \\
\text { A6 Projetos e análises sólidos. As avaliações devem } \\
\text { empregar projetos e análises tecnicamente adequados, } \\
\text { adequados para os fins da avaliação. } \\
\text { A7 Raciocínio explícito da avaliação. O raciocínio da } \\
\text { avaliação deve levar informações e análises a descobertas, }\end{array}$ \\
\hline
\end{tabular}




\begin{tabular}{|c|c|}
\hline & $\begin{array}{l}\text { interpretações, conclusões e julgamentos de forma clara e } \\
\text { completamente documentada. } \\
\text { A8 Comunicação e relatórios. As comunicações da } \\
\text { avaliação devem ter escopo adequado e proteger-se de } \\
\text { conceitos errôneos, preconceitos, distorções e erros. }\end{array}$ \\
\hline \multicolumn{2}{|c|}{ Padrões de avaliação da responsabilidade } \\
\hline $\begin{array}{l}\text { Os padrões avaliação da } \\
\text { responsabilidade da avaliação } \\
\text { incentivam a documentação } \\
\text { adequada das avaliações e uma } \\
\text { perspectiva meta-avaliadora } \\
\text { focada na melhoria e prestação } \\
\text { de contas dos processos e } \\
\text { produtos de avaliação. }\end{array}$ & $\begin{array}{l}\text { E1 Documentação da avaliação. As avaliações devem } \\
\text { documentar completamente seus objetivos negociados e os } \\
\text { projetos, procedimentos, dados e resultados } \\
\text { implementados. } \\
\text { E2 Avaliação interna de meta-avaliação. Os avaliadores } \\
\text { devem usar estes e outros padrões aplicáveis para } \\
\text { examinar a responsabilidade do desenho da avaliação, } \\
\text { procedimentos empregados, informações coletadas e } \\
\text { resultados. } \\
\text { E3 Os patrocinadores, clientes, avaliadores e outras partes } \\
\text { interessadas no programa de meta-avaliação externa } \\
\text { devem incentivar a realização de meta-avaliações externas } \\
\text { usando esses e outros padrões aplicáveis. }\end{array}$ \\
\hline
\end{tabular}

Fonte: adaptado de JCEE (2020).

\section{Procedimentos metodológicos}

Com o propósito de averiguar se há algum tipo de preparação para os membros da CPA que os capacite para a atividade avaliativa e se os membros consideram necessária essa capacitação, foram entrevistadas as presidentes das CPAs das faculdades Senac Florianópolis e Palhoça. Considerando que o Senac possui dez faculdades no Estado de Santa Catarina, optou-se pela grande Florianópolis pela proximidade entre as instituições e pelo acesso aos interlocutores da presente pesquisa, ou seja, uma amostra por conveniência.

O intuito deste estudo de caso foi também verificar, por meio da entrevista junto às presidentes das CPAs, se é feita a análise posterior do relatório da autoavaliação institucional encaminhado anualmente ao $\mathrm{MEC}$ e se é realizado algum tipo de metaavaliação a partir desta análise

Além disso a entrevista possibilitou apresentar os atributos e critérios do Join Committee on Standards Educational Evaluation - JCSEE - selecionados para esta pesquisa, a fim de questionar suas opiniões acerca da possibilidade de aplicação destes como parte de um instrumento de meta-avaliação, considerando as especificidades da autoavaliação institucional.

Foi elaborado um questionário com dez perguntas abertas, enviado por e-mail às respondentes, as quais responderam também por e-mail. Foram feitas videoconferências com ambas para discutir sobre as respostas. As respostas às questões abertas foram registradas conforme retornado no questionário pelas respondentes. Trata-se, portanto, de uma pesquisa qualitativa, pois analisa os resultados da opinião dos pesquisados sobre os assuntos abordados.

Além do estudo qualitativo foi realizada pesquisa documental, com a análise dos relatórios anuais da CPA de cada uma das faculdades: relatórios finais do primeiro ciclo avaliativo (2017) e relatórios parciais do ciclo vigente (2018). De acordo com Godoy 
(1995) a pesquisa qualitativa não precisa ser necessariamente apresentada como uma proposta rígida e estruturada, pois, permite aos pesquisadores a busca por novos trabalhos, trazendo novos enforques. A pesquisa documental, portanto, pode ser considerada de caráter inovador, com importantes fontes de dados que contribuem para os estudos de alguns temas.

Desta forma a análise dos relatórios buscou identificar a metodologia e os resultados da pesquisa aplicada pela comissão para diagnosticar o desempenho da autoavaliação à luz das dimensões estabelecidas pela legislação.

\section{Apresentação e análise dos resultados}

As duas entrevistadas são presidentes das CPAs das Faculdades Senac de Florianópolis (R1) e de Palhoça (R2). As quatro primeiras perguntas do questionário dizem respeito à experiência e à capacitação das entrevistadas para atuação na CPA.

A primeira questão refere-se ao tempo em que as entrevistadas atuam com a CPA. R1 participa de CPA desde 2011, assumindo a presidência da comissão em 2019. Já R2 é membro desde 2016, assumindo a presidência em 2017.

Quanto à experiência anterior das entrevistadas com esse tipo de comissão foi questionado se já tiveram participações anteriores ambas participavam como docentes, somente como respondentes das pesquisas, quando solicitadas.

A questão três verificou se alguma das entrevistadas teve algum tipo de capacitação ou orientação para o desenvolvimento deste processo. Ambas relatam que não:

R1 - Não. Em 2011 o processo era executado pela Coordenação do Núcleo de Educação Superior da Faculdade; os membros contribuíam apenas com a análise dos resultados da autoavaliação.

R2 - Não especificamente, porém, na época a coordenadora da graduação fazia reuniões e destas já orientava sobre o trabalho, e também o Senac Departamento Regional, proporciona documentos orientativos, que respalda sobre tudo que é inerente aos cursos executados pela instituição, temos disponível na intranet material de apoio, modelo de relatório, arte de divulgação para campanha da autoavaliação, suporte da equipe pedagógica e administrativa.

$\mathrm{Na}$ sequência a entrevista buscou saber de que forma as respondentes se capacitaram para atuar como avaliadoras na CPA:

R1 - Estudando sobre: Sinaes, Atribuições da CPA, Dimensões avaliadas na Avaliação Institucional, Roteiro de Autoavaliação Institucional, Análise de Avaliação Institucional de outras IES.

R2 - Através das reuniões e capacitações organizadas pela unidade/NDS, material disponível na intranet e internet, participei de um curso on-line do Inep.

A partir destas respostas é possível analisar que não há uma capacitação estruturada para os membros da CPA nas faculdades Senac de Florianópolis. No entanto, para que a comissão possa cumprir com suas atribuições, atingindo os objetivos da autoavaliação, este é um requisito fundamental. 
O conjunto de perguntas de cinco a sete do questionário abordou a elaboração do relatório de autoavaliação pela CPA e o tratamento das informações depois de publicado, incluindo a verificação da existência de algum tipo de meta-avaliação do próprio relatório, com a finalidade de aprimoramento do documento. Segundo R1 as ações da CPA na elaboração do relatório consistem em,

após a aplicação dos questionários, são criadas planilhas com o quantitativo do resultado dessa aplicação, indicando também as potencialidades e fragilidades apontadas por cada público e registrando os comentários apresentados na pesquisa. As análises por curso (alunos e professores) são realizadas pelos NDE's e dos demais públicos: corpo técnico, comunidade externa e egressos) é analisada pela equipe da CPA. Destas análises, são indicadas proposições de melhorias que são compiladas na ferramenta $5 \mathrm{~W} 2 \mathrm{H}$ e apresentada para à direção.

Na Faculdade Senac Palhoça o processo é bem similar: "Após aplicação da autoavaliação é compilado os dados e desenvolvido o relatório, que normalmente conta com a participação dos membros da CPA e também do NDE" (R2).

Embora ambas as respondentes tenham afirmado não haver nenhum tipo de avaliação da autoavaliação institucional, meta-avaliação, respondendo à questão seis R1 mencionou que "estamos sempre repensando o processo para melhorá-lo. No entanto, esse processo de avaliação, neste momento, não é baseado em critérios definidos/estabelecidos".

Tanto R1, quanto R2, consideraram importante que haja algum tipo de metaavaliação para o processo de autoavaliação institucional. A questão sete foi direcionada à avaliação do relatório publicado pela CPA, no entanto, a R1 apontou que

R1 - Penso que é necessário analisar todo o processo que resulta na Autoavaliação Institucional, da organização do questionário à elaboração final do relatório. Apenas a análise do relatório da Autoavaliação Institucional pode não identificar falhas no processo, impedindo, dessa forma, ajustes ao longo do processo.

Considerando as respostas deste conjunto de perguntas pode-se verificar que é essencial a participação dos membros da CPA e do Núcleo Docente Estruturante no processo de elaboração do relatório. No entanto, ainda que se possa visualizar sua intenção em realizar um bom trabalho, a falta de sistematização de um processo de metaavaliação, com fundamentos teóricos, faz com que as comissões acabem entrando num ciclo, sem muitas possibilidades de apontamentos de melhoria no processo por não haver um processo de meta-avaliação institucionalizada.

O último grupo de perguntas foi direcionado ao conceito de meta-avaliação e à sua aplicabilidade em relação aos processos avaliativos da CPA.

A questão oito tratou sobre a possibilidade de estabelecer atributos e critérios que contribuam na elaboração de um processo de meta-avaliação. Nesse sentido a R1 afirmou que sim, desde que estes atributos e critérios possam ser aplicados em todo o processo e não somente ao relatório final da autoavaliação. Já a R2 apontou que a "utilidade da meta-avaliação é de verificar a qualidade do processo avaliativo aplicado, oferecendo subsídios para revisão e aprimoramento da própria avaliação". 
Na pergunta nove foi questionado se as respondentes teriam alguma sugestão de metodologia para a elaboração de um instrumento padrão de meta-avaliação para autoavaliação institucional.

Para R1 a meta-avaliação deve fazer parte de todo o processo de autoavaliação e "deve analisar todas as etapas que compõem o processo" (R1).

Nessa questão R2 apresentou uma proposta de encaminhamento por e-mail, para organização das informações:

R2 - Acredito que a partir do instrumento de meta-avaliação padrão elaborado, este deva ser apresentado por meio de e-mail contendo objetivos e instruções de preenchimento, disponibilizado para direção, coordenadores de núcleos, cursos e membros da CPA solicitando aos participantes que se posicionassem em relação às informações e que indicassem seu grau de concordância para cada um dos itens apresentados, assim teremos na instituição Senac SC um instrumento participativo.

$\mathrm{Na}$ última pergunta do questionário foi apresentado quadro abaixo com dois dos atributos formulados pelo JCSEE e seus respectivos critérios, solicitando às respondentes se acreditam que é possível elaborar um instrumento padrão com a finalidade de estabelecer um processo de meta-avaliação para a autoavaliação institucional no Senac/SC.

Quadro 2 -

Atributos e critérios propostos para meta-avaliação da autoavaliação.

\begin{tabular}{|c|c|l|}
\hline Atributos & \multicolumn{3}{|c|}{ Critérios } \\
\hline \multirow{4}{*}{ Precisão } & A1 & Conclusões e decisões justificadas \\
\cline { 2 - 3 } & A2 & Informações válidas \\
\cline { 2 - 3 } & A3 & Procedimentos de avaliação de informações confiáveis \\
\cline { 2 - 3 } & A4 & Descrições explícitas do programa e do contexto \\
\cline { 2 - 3 } & A5 & Avaliações de gerenciamento de informações \\
\cline { 2 - 3 } & A6 & Projetos e análises sólidos \\
\cline { 2 - 3 } & A7 & Raciocínio explícito da avaliação \\
\cline { 2 - 3 } & A8 & Comunicação e relatórios \\
\hline \multirow{4}{*}{ Responsabilização } & R1 & Documentação da Avaliação \\
\cline { 2 - 3 } & R2 & Meta-avaliação Interna \\
\cline { 2 - 3 } & R3 & Meta-avaliação Externa \\
\hline
\end{tabular}

Fonte: adaptado de JCSEE (2020).

Observando o quadro acima as respondentes concordam que tais atributos possam contribuir para a elaboração do referido instrumento, entretanto, a R1 apresenta um ponto de vista interessante:

R1 - Caso seja de responsabilidade da Comissão penso que os atributos e critérios devem possibilitar a análise de todo o processo de autoavaliação, como já descrito em outros itens. Se essa análise for realizada por alguém que não participa do processo, a meta-avaliação ocorrerá com base no relatório, assim, os atributos e critérios aqui definidos, em uma primeira análise, permitem a definição do instrumento. 
A partir desta observação a R1 abordou questões a serem discutidas ao elaborar um instrumento padrão para meta-avaliação da autoavaliação: se for de responsabilidade da CPA é importante que este processo contemple todas as etapas da autoavaliação e não apenas o relatório, neste caso, seria prudente rever os atributos para que o instrumento possa trazer as melhorias para o processo como um todo. Caso se opte por atribuir a terceiros corre-se o risco de os mesmos focarem apenas nos documentos e, ainda que os atributos citados possam dar conta de avaliar todo o relatório, não significa que este processo possa trazer melhorias em todas as etapas da autoavaliação.

Além da entrevista também se analisou os relatórios publicados pelas CPAs das Faculdades Senac de Florianópolis, considerando o final do ciclo 2015-2017, relatório geral, e o início do ciclo 2018-2020, relatório parcial. Observou-se que ambas comissões se embasam na Nota técnica Inep/Daes/Conaes n. 065/2014, que estabelece o roteiro para relatório de autoavaliação institucional. Além disso, analisando os demais documentos institucionais do Senac/SC, observou-se também, conforme citado pelas entrevistadas, que há um documento emitido pela mantenedora intitulado Regulamento da CPA e da autoavaliação das Faculdades Senac/SC, o qual determina a composição das comissões, as competências e o processo de coleta e análise de dados das pesquisas da CPA.

Todos os relatórios apresentam as dimensões estabelecidas pelo Sinaes, as quais são avaliadas pelos diversos públicos participantes da pesquisa, e todos apresentam o plano de ação a partir das fragilidades observadas pelos resultados, assim como os apontamentos do que foi desenvolvido pelos planos de ação dos relatórios anteriores.

Assim sendo, pode-se considerar tanto os resultados das entrevistas, quanto os relatórios de autoavaliação das faculdades pesquisadas elementos importantes na elaboração de um instrumento padrão de meta-avaliação para a instituição.

\section{Considerações finais}

A autoavaliação institucional, conduzida pela CPA, tem o propósito de possibilitar autoconhecimento institucional e deve ser aplicada desde o nascimento da instituição de forma permanente e autônoma, expressando a situação da instituição e primando por apontar melhorias que levem à excelência dos serviços prestados à sociedade (Hajj et al, 2018).

A avaliação, por si só, é uma ação complexa e requer uma série de princípios e concepções para que obtenha o sucesso desejado. Se o objetivo da avaliação não for claro e se todos os envolvidos não estiverem imersos no processo, a avaliação pode chegar a resultados insignificantes ou trazer respostas sem sentido. Ou seja, por mais que se tenha boa intenção ao realizar uma avaliação, se não houver competência para tanto, poderão surgir conflitos no processo avaliativo. "Em síntese, eles poderão se caracterizar pela deterioração da qualidade da avaliação, pela falha na identificação e no envolvimento dos stakeholders, pela inadequada formação do avaliador, ou pela não utilização plena dos resultados". (Firme, Letichevsky, 2010).

O resultado desta pesquisa mostrou que, embora as CPAs se empenhem em fazer um bom trabalho, não há qualquer capacitação sistematizada, no Senac/SC, para esse tipo de avaliação. 
A ausência de familiaridade da comunidade acadêmica com teorias, metodologias e práticas de avaliação favorece 0 desencontro entre objetivos propostos, estratégias metodológicas empregadas e resultados alcançados pela CPA (Gomes; Silva, 2011). Para tanto o processo de meta-avaliação se torna imprescindível no sentido de constatar sua eficácia e eficiência, uma vez que avaliar a avaliação abre espaço para discussão sobre os encaminhamentos da própria comissão e o envolvimento dos stakeholders.

Segundo os resultados desta pesquisa pode-se concluir que é possível criar um instrumento de meta-avaliação para a autoavaliação institucional. Este é um tema que deixamos como sugestão para trabalhos futuros, considerando que o processo formal de autoavaliação é estabelecido pela legislação educacional, orientado por órgãos competentes e desempenhado sempre pelas CPAs.

\section{Referências}

BRASIL. Constituição da República Federativa do Brasil. Brasília: Senado Federal, 1988 BRASIL. Lei n. 9.394, de 20 de dezembro de 1996: estabelece as diretrizes e bases da educação nacional. Brasília: Senado Federal, 1996.

BRASIL. Lei n. 10.861, de 14 de abril de 2004: institui o Sistema Nacional de Avaliação da Educação Superior - Sinaes e dá outras providências. Brasília: Senado Federal 2004.

BRASIL. Nota técnica Inep/Daes/Conaes n. 65/2014: roteiro para relatório de autoavaliação institucional. Brasília: Inep, 2014.

HAJJ, Zaina El; COSTA, Barbara Regina Lopes; ARAÚJO, Richard Medeiros. O papel das comissões próprias de avaliação sobre os atos regulatórios: um olhar da gestão universitária. Meta: Avaliação, Rio de Janeiro, v. 10, n. 28, 2018, p. 75-105.

DAVOK, Deise Fries. Modelo de meta-avaliação de processos de avaliação da qualidade de cursos de graduação. Florianópolis: UFSC, 2006. 274f. Tese (Doutorado em Engenharia de Produção). Universidade Federal de Santa Catarina.

FIRME, Thereza Penna; LETICHEVSKY, Ana Carolina. O desenvolvimento da capacidade de avaliação no século 21: enfrentando o desafio através da meta-avaliação. Meta: Avaliação, Rio de Janeiro, v. 2, n. 5, 2010, p. 180-195.

GALVÃO, Maria Cecília Alves; PEREIRA, Hércules; ANNUNZIATA NETO, Luiz. A metaavaliação como instrumento de qualificação do processo de autoavaliação em uma instituição de ensino superior no Rio de Janeiro. CONGRESSO IBERO-AMERICANO DE POLÍTICA E ADMINISTRAÇÃO DA EDUCAÇÃO, 1, 2014. Anais ... Porto: Anpae, 2014, p. 1-18.

GIMENES, Nelson Antonio Simão. Estudo meta-avaliativo do processo de autoavaliação em uma instituição de educação superior no Brasil. Estudos em Avaliação Educacional, São Paulo, v. 8, n. 37, 2007, p. 217-243.

GODOY, Arilda Schmidt. Pesquisa qualitativa: tipos fundamentais. Revista de Administração de Empresas, São Paulo, v. 35, n. 3, 1995, p. 20-29.

INEP. Sistema nacional de avaliação da educação superior: bases para uma nova proposta de avaliação da Educação Superior. Brasília: Inep, 2004.

INEP. Anais dos seminários regionais sobre autoavaliação institucional e comissões próprias de avaliação (CPA). Brasília: Inep, 2015. 
JCSEE. Joint committee on standards for educational evaluation. 2020. Disponível em: https://jicsee.org/. Acesso em: 11 nov. 2020.

JCSEE. The program evaluation standards: a guide for evaluators and evaluation users: aguide for evaluators and evaluation users. Thousands Oaks, CA: Sage, 2011.

PINTO, Rodrigo Serpa. Meta-avaliação: uma década do processo de avaliação institucional. Florianópolis: UFSC, 2015. 269f. Tese (Doutorado em Administração). Universidade Federal de Santa Catarina.

SANTOS, Eliane Gomes dos; SADALA, Maria da Glória Schwab; BORGES, Sônia Xavier de Almeida. Avaliação institucional: por que os atores silenciam? Educação e Realidade, Porto Alegre, v. 37, n. 2, 2012, p. 551-568.

SILVA, Assis Leão da; GOMES, Alfredo Macedo. Avaliação institucional no contexto do sinaes: a cpa em questão. Avaliação: Revista da Avaliação da Educação Superior, Campinas, v. 16, n. 3, ano 2011, p. 573-601.

SORDI, Mara Regina Lemes de. Comissão própria de avaliação (CPA): similaridades e dessemelhança no uso da estratégia na educação superior e em escolas do ensino fundamental. Avaliação: Revista da Avaliação da Educação Superior, Campinas v. 16, n. 3, 2011, p. 603-617.

SOUZA, Valesca Rodrigues de; KIPNIS, Bernardo. A autoavaliação institucional na elaboração da identidade do Instituto Federal do Norte de Minas Gerais. SIMPÓSIO AVALIAÇÃO DA EDUCAÇÃO SUPERIOR, 2, 2016. Anais ... Porto Alegre: Ufrgs, 2016.

STUFFLEBEAM, Daniel Leroy. The methodology of metaevaluation as reflected in metaevaluations by the Western Michigan University Evaluation Center. Journal Of Personnel Evaluation In Education, Norwell, M.A, v. 14, n. 1, 2001, p. 95-125.

Melissa Maria de Souza Zimmermann é estudante no Programa de Pós-Graduação em Administração Universitária da Universidade Federal de Santa Catarina.

Orcid: https://orcid.org/0000-0003-3674-2391.

Endereço: Rua Gisela, 1257 - 88110-110 - São José - SC - Brasil.

E-mail: melissazm@gmail.com.

Lourdes Alves é professora no Programa de Pós-Graduação em Administração Universitária da Universidade Federal de Santa Catarina.

Orcid: https://orcid.org/0000-0003-1518-8643.

Endereço: Avenida Presidente Nereu Ramos, 1100/305 - 88101-410 - São José - SC

- Brasil.

E-mail: loual@lourdesalves.com.br.

Critérios de autoria: Melissa realizou a concepção, execução, análise, interpretação e redação. Lourdes Alves realizou a supervisão do planejamento e execução da atividade de pesquisa.

Recebido em 21 de maio de 2021.

Aceito em 23 de julho de 2021.

(c) (i) (\$) $\odot$

Regae: Rev. Gest. Aval. Educ.

Santa Maria

v. 10

ก. 19

e65887, p. 1-13

2021 